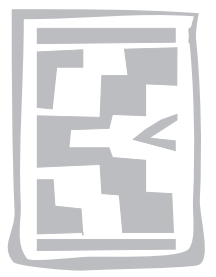

\title{
Some preliminary observations on the susceptibility and resistance of different cattle breeds to Theileria parva infection
}

\author{
S.G. NDUNGU, P.N. NGUMI, S.K. MBOGO, T.T. DOLAN, J.J. MUTUGI and A.S. YOUNG \\ Kenya Agricultural Research Institute, National Research Centre, Muguga, P.O. Box 32, Kikuyu, Kenya
}

\begin{abstract}
NDUNGU, S.G., NGUMI, P.N., MBOGO, S.K., DOLAN, T.T., MUTUGI, J.J. \& YOUNG, A.S. 2005. Some preliminary observations on the susceptibility and resistance of different cattle breeds to Theileria parva infection. Onderstepoort Journal of Veterinary Research, 72:7-11

Theileria parva-naïve Friesian (Bos taurus), Boran (Bos indicus) and Maasai Zebu steers (B. indicus) were infected with a $T$. parva sporozoite stabilate dose which had previously been shown to induce an estimated $50 \%$ mortality rate in Boran cattle. All the cattle developed patent infections with no significant differences in the length of the prepatent period to development of macroschizonts $(P>$ $0.05)$ between the three groups. Clinical theileriosis occurred in all eight the Friesians $(100 \%)$, five out of nine Borans $(55.6 \%)$ and two out of five Zebus (40\%). Three of the Friesians $(37.5 \%)$, and two of the Borans $(22.2 \%)$ died of theileriosis. The different cattle types were equally susceptible to the infective dose used as indicated by the length of the prepatent periods, but there was a marked difference in their development of clinical theileriosis. The gradation in resistance to disease confirms the findings of earlier less critical studies and identifies these cattle breeds as suitable for investigations into the mechanisms of resistance to theileriosis.
\end{abstract}

Keywords: Boran, cattle, Friesian, resistance, susceptibility, Theileria parva, theileriosis

\section{INTRODUCTION}

Cattle from Theileria parva-endemic areas in East Africa are reported to show a high degree of natural resistance to theileriosis (Barnett 1968; Young 1981; Moll, Lodhing \& Young 1984; Moll, Lodhing, Young \& Leitcth 1986). Stobbs (1966) showed that Boran cattle (Bos indicus) were more susceptible to theileriosis in an endemic area than indigenous Zebu cattle (B. indicus) or the offspring of the introduced and indigenous cattle. The susceptibility of $B o s$ taurus and $B$. indicus breeds of cattle to experimental $T$. parva infection using infected ticks

Accepted for publication 28 September 2004-Editor
(Barnett 1968), sporozoite stabilate (Radley 1978) and $T$. parva schizont-infected lymphoblastoid cell lines (Dolan, Njuguna \& Stagg 1982) has been reported to be different with the Boran/Zebu cattle being less susceptible to infection and the development of clinical theileriosis.

In this experiment a $T$. parva sporozoite stabilate whose infectivity and pathogenicity had been determined by titration in Boran cattle (Dolan, Young, Losos, McMillan, Minder \& Soulsby 1984) was used to compare the susceptibility of different cattle types. Zebu cattle from an endemic area, and Boran and Friesian cattle from non-endemic areas, all of which had been raised free of theileriosis, were challenged with an estimated $50 \%$ lethal dose $\left(\mathrm{LD}_{50}\right)$ of $T$. parva sporozoite stabilate for Boran cattle to determine 
their relative susceptibility to infection and resistance to the development of disease.

\section{MATERIALS AND METHODS}

\section{Experimental cattle}

Five pregnant Maasai Zebu (B. indicus) cows were purchased from the Trans-Mara area of Kenya and transported to the National Veterinary Research Centre (NVRC), Muguga. Calves born of these cows were used in the trial. This breed has been described by Mason \& Maule (1960). The Trans-Mara area is endemic for theileriosis as has been previously reported (Moll et al. 1984, 1986). These calves were allowed to suckle colostrum and were subject to a strict tick control programme in which they were treated once a week. They were used in the experiment when they were between 15 and 18 months old. Before the commencement of the trial, they were tested negative for $T$. parva antibodies, using the indirect fluorescent antibody test (IFAT) on serum samples with a cell culture of $T$. parva schizonts as antigen (Burridge \& Kimber 1972). Eight Friesian (B. taurus) and Boran (B. indicus) cattle all approximately 18 months old were purchased from farms in Kenya where strict tick control was practised and which were negative for $T$. parva antibodies with the IFAT prior to the experiment. The Boran, a beef breed originating from Northern Kenya and Southern Ethiopia, has been defined by Mason \& Maule (1960).

All animals were dewormed using a mixture of oxyclosamide and levamisole hydrochloride (Nilzan, ICI England) and treated with imidocarb dipropionate (Imizol, Wellcome, England) chemoprophylactically against babesiosis and anaplasmosis before infection. The cattle were kept together in a partially covered yard under tick-free conditions.

\section{Theileria parasites}

The sporozoite stabilate used was the T. parva (Muguga stabilate 147) prepared according to the method of Radley (1978) and Cunningham, Brown, Burridge \& Purnell (1973), and characterised in detail by Dolan et al. (1984). The stabilate was diluted with Eagle's Minimum Essential Medium (MEM) with 3.5 bovine plasma albumin (BPA) weight per volume $(\mathrm{w} / \mathrm{v})$ and $7.5 \%$ glycerol volume per volume $(\mathrm{v} / \mathrm{v})$ to $10^{-1.3}$ (1 in 20) which is estimated to be the $\mathrm{LD}_{50}$ when used to infect Boran cattle. Each of the cattle was infected with $1 \mathrm{ml}$ of the diluted stabilate subcutaneously in front and immediately below the left ear.

\section{Parameters recorded}

\section{Temperatures}

Rectal temperatures were recorded daily between 7.30 and 8.00 from the day of inoculation (day 0 ) to the time of death or day 28 after inoculation whichever came earlier. A temperature of $39.5^{\circ} \mathrm{C}$ or above associated with Theilerial schizont parasitosis was considered to be a febrile response.

\section{Haematology}

The cattle were bled from the jugular veins for haematology before inoculation and thereafter, three times a week until death or day 28 . The sodium salt of EDTA was used as the anti-coagulant. White blood cell counts (WBC) and red blood cell counts (RBC) were determined using an electronic particle counter (Model ZN; Coulter Electronic Inc., Florida, USA). The haemoglobin values were determined using a haemoglobinometer (Coulter Electronic Inc., Florida, USA). Packed cell volumes (PCV) were estimated using the microhaematocrit method.

\section{Serology}

Blood for serology was taken from the jugular veins of cattle prior to the start of the experiment and once a week after inoculation until day 35. Antibody titres were determined using the IFAT. A titre of $1: 40$ or above was considered positive.

\section{Parasitological observations}

Needle biopsies were taken daily from the local drainage lymph node (left parotid) from day 5 to day 28 after inoculation. The biopsies were smeared on glass microscope slides, and the smears fixed in methanol, stained with Giemsa's stain and examined under the microscope for theilerial schizonts. Once schizonts were observed, biopsy smears were made from the contralateral prescapular lymph nodes and examined to determine the degree of dissemination of the parasites. Blood smears were prepared from tail-tip blood once schizonts in the lymph node smears were detected and were similarly stained and examined for the presence of intra-erythrocytic piroplasms.

\section{Definitions}

The day of recovery was considered to be the day after the last schizonts were detected. A mild reaction was considered to be one where low numbers of schizonts (less than one per microscope field at 
$1000 \times$ magnification) occurred, associated with no or transient fever lasting for 1 or 2 days. A severe reaction was considered to be an infection where schizonts were plentiful (one or more schizonts per microscope field at $1000 \times$ magnification), a prolonged fever was evident and the animal recovered. In a very severe reaction, schizonts were plentiful, fever was prolonged and the animal died or was euthanased in extremis. The main factors considered were susceptibility to infection and susceptibility to disease. In this paper establishment of infection is termed susceptibility while susceptibility together with the ability to clear the infection is referred to as resistance.

\section{Data analysis}

Statistical analysis between the three groups was performed using the non-parametric Kruskal-Wallis test. Statistical analysis between any two groups was performed using the Mann-Whitney $U$ test.

\section{RESULTS}

All cattle became infected and developed a schizont parasitosis. The theilerial reactions of individual cattle are summarized in Table 1.

There were no significant differences $(P>0.05)$ in the prepatent period to schizonts or the time to fever $(P>0.05)$ between the groups. However, all the Friesians (100\%), five of the nine Borans (55\%) and two out of five Zebus (40\%) developed a febrile response.

There were marked differences in disease reactions. The five Zebus, four of the Borans and one of the Friesians had mild reactions. Three of the Borans and three of the Friesians had severe reactions while two of the Borans and four of the Friesians had very severe reactions. In those that did recover there was no significant difference in the time to recovery between the three groups $(P>$ 0.05 ) or between any two groups [Friesians/Borans

TABLE 1 Theilerial reaction of three cattle breeds infected with $10^{-1.3}$ dilution of $T$. parva (parva) (Muguga) stabilate

\begin{tabular}{|c|c|c|c|c|c|c|}
\hline Cattle type & Animal no. & $\begin{array}{l}\text { Macroschizont } \\
\text { appearance }\end{array}$ & $\begin{array}{l}\text { Temperature } \\
\text { reaction }\end{array}$ & Recovery & Death & $\begin{array}{l}\text { Classification } \\
\text { of reaction }\end{array}$ \\
\hline \multirow[t]{2}{*}{$\begin{array}{l}\text { Friesian } \\
\text { Bos taurus }\end{array}$} & $\begin{array}{l}44 \\
45 \\
46 \\
48 \\
49 \\
51 \\
52 \\
53\end{array}$ & $\begin{array}{r}10 \\
10 \\
10 \\
9 \\
13 \\
10 \\
9 \\
11\end{array}$ & $\begin{array}{l}12 \\
14 \\
13 \\
13 \\
14 \\
15 \\
13 \\
13\end{array}$ & $\begin{array}{r}17 \\
- \\
18 \\
24 \\
27 \\
21 \\
- \\
-\end{array}$ & $\begin{array}{r}- \\
22 \\
- \\
- \\
- \\
- \\
17 \\
19\end{array}$ & $\begin{array}{l}M \\
\text { VS } \\
S \\
S \\
\text { VS } \\
S \\
\text { VS } \\
\text { VS }\end{array}$ \\
\hline & Mean $\pm S D$ & $10.3 \pm 1.3$ & $13.4 \pm 0.9$ & $21.4 \pm 0.9$ & $19.3 \pm 2.5$ & M1/S3/VS4 \\
\hline \multirow[t]{2}{*}{$\begin{array}{l}\text { Boran } \\
\text { Bos taurus }\end{array}$} & $\begin{array}{l}869 \\
871 \\
895 \\
876 \\
885 \\
886 \\
888 \\
890 \\
892\end{array}$ & $\begin{array}{r}7 \\
10 \\
10 \\
9 \\
9 \\
9 \\
10 \\
11 \\
7\end{array}$ & $\begin{array}{r}15 \\
- \\
- \\
12 \\
13 \\
12 \\
- \\
- \\
15\end{array}$ & $\begin{array}{r}20 \\
19 \\
20 \\
- \\
24 \\
21 \\
16 \\
- \\
19\end{array}$ & $\begin{array}{r}- \\
- \\
- \\
22 \\
- \\
- \\
- \\
22 \\
-\end{array}$ & $\begin{array}{l}S \\
M \\
M \\
V S \\
S \\
M \\
M \\
V S \\
\text { VS }\end{array}$ \\
\hline & Mean $\pm S D$ & $9.1 \pm 1.4$ & $13.4 \pm 1.5$ & $19.6 \pm 2.4$ & 22 & M4/S3/VS2 \\
\hline \multirow[t]{2}{*}{$\begin{array}{l}\text { Zebu } \\
\text { Bos indicus }\end{array}$} & $\begin{array}{l}553 \\
563 \\
564 \\
664 \\
767\end{array}$ & $\begin{array}{r}10 \\
11 \\
8 \\
9 \\
12\end{array}$ & $\begin{array}{r}- \\
- \\
- \\
13 \\
15\end{array}$ & $\begin{array}{l}13 \\
15 \\
16 \\
15 \\
23\end{array}$ & $\begin{array}{l}- \\
- \\
- \\
- \\
-\end{array}$ & $\begin{array}{l}M \\
M \\
M \\
M \\
M\end{array}$ \\
\hline & Mean \pm SD & $10.0 \pm 1.6$ & 14 & $16.4 \pm 3.8$ & & M5 \\
\hline
\end{tabular}

$\mathrm{M}=$ Mild reaction; $\mathrm{S}$ = Severe reaction; $\mathrm{VS}$ = Very severe reaction; SD = Standard deviation 
$(P>0.05)$, Friesians/Zebus, $(P>0.05)$ and Borans/ Zebus $(P>0.05)]$.

The most marked change noted in the haematological parameters was the depression of the leukocyte counts which was directly related to the severity of disease. There was no significant difference in the degree of leukocyte count depressions between the three cattle groups. The depression was least in the Zebu group.

All surviving cattle showed an antibody response to $T$. parva schizont antigen but the differences in antibody titres among the different cattle types were not statistically significant.

\section{DISCUSSION}

An unexpected finding in this experiment was that the three cattle breeds showed no significant differences in susceptibility, which, in this case, was judged by the length of the prepatent period which ended when macroschizonts were detected in the lymph node smears. Theileria parva infection in cattle is dose dependent in its severity. The more sporozoites inoculated the more acute the course of disease (Jarret, Crighton \& Pirie 1969; Radley, Brown, Burridge, Cunningham, Pierce \& Purnell 1974; Morrison, Buscher, Murray, Emery, Masake, Cook \& Wells 1981; Dolan et al. 1984; Mutugi, Young, Maritim, Ndungu, Stagg, Grootenhuis \& Leitch 1988). The fact that length of the prepatent period was the same in the three breeds means that approximately similar numbers of sporozoite-infected target cells and that the schizont-infected cells divided at a similar rate (Stagg, Young, Leitch \& Dolan 1983). An alternative method of comparing the susceptibility of the cattle breeds would have been to compare the $50 \%$ infectious dose $\left(\mathrm{ID}_{50}\right)$ in the different breeds. Radley (1978) found indications that Friesian and Boran breed cattle had different $I D_{50}$ with Friesians showing a higher susceptibility. However, the comparison made in this study was more practical since the minimum quantum of infection delivered by a tick in nature is probably of the order of that used in the present experiment. Therefore, the present study is a better representation of the natural conditions than evaluation of the $\mathrm{ID}_{50}$.

A marked difference, however, occurred between the breeds in the development of clinical disease. We have associated this observation with the different degrees of resistance between the breeds. The Zebu cattle showed a higher degree of resistance than either the Boran or Friesian cattle since more became clinically sick while theileriosis and deaths occurred in the other groups. Barnett \& Bailey (1955) and Barnett (1968) reported that a high degree of resistance to theileriosis occurred in cattle from endemic areas which was confirmed in the field by Moll et al. (1984, 1986). Stobbs (1966) demonstrated clearly the inheritance of resistance to theileriosis when he introduced Boran cattle into an ECF-endemic area of Uganda and cross-bred them with local Zebu cattle. The cross-bred cattle were intermediate in their resistance to theileriosis compared to their parental type. This study is considered an improvement over those of Barnett \& Bailey (1955), Barnett (1968) and Stobbs (1966) in that a reproducible challenge of diluted stabilate was used while in the other studies natural or experimental challenge with feeding ticks was used. In the latter the success rate of the feeding of the variably infected ticks could have influenced their results. The mechanism of resistance to $T$. parva infection is not known but it is likely to be due to a more effective cell mediated response in the Zebu cattle than in the other breeds (Baldwin, Malu, Kinuthia, Conrad \& Grootenhuis 1986). It is imperative that the mechanism of resistance be investigated further. The exploitation of greater resistance to theileriosis in cross-bred cattle might prove to be an important strategy in cattle improvement programmes in East Africa if this resistance trait could be defined further.

\section{ACKNOWLEDGEMENTS}

The ideas provided by members of the Protozoology Division, National Veterinary Research Centre are gratefully acknowledged. The research work undertaken was funded by a joint Kenyan and British government project entitled "Application and monitoring of infection and treatment method of immunisation against theileriosis in the field". This paper is published with kind permission of the Directors of the Kenya Agricultural Research Institute and the International Livestock Research Institute.

\section{REFERENCES}

BALDWIN, C.L., MALU, M.N., KINUTHIA, S.W., CONRAD, P.A. \& GROOTENHUIS, J.G. 1986. Comparative analysis of infection and transformation of lymphocytes from African buffalo and Boran cattle with Theileria parva parva and Theileria parva lawrencei. Infection and Immunity, 53:186-191.

BARNETT, S.F. \& BAILEY, K.P. 1955. The susceptibility of Zebu calves to ECF under experimental conditions at Muguga. East African Veterinary Research Organisation Annual Report 1954-1955, East African High Commission, Nairobi, Kenya. 
BARNETT, S.F. 1968. Theileriosis, in Infectious blood diseases of man and animals, edited by D. Weinman \& M. Ristic, Vol. 2. New York: Academic Press.

BURRIDGE, M.M. \& KIMBER, C.D. 1972. The indirect fluorescent antibody test for East Coast fever (Theileria parva infection of cattle). Evaluation of a cell culture schizont antigen. Research in Veterinary Science, 13:451-455.

CUNNINGHAM, M.P., BROWN, C.G.D., BURRIDGE, M.J. \& PURNELL, R.E. 1973. Cryopreservation of infective particles of Theileria parva. International Journal for Parasitology, 3:583-587.

DOLAN, T.T. \& McHARDY, N. 1978. The chemotherapy of experimental T. parva infection, in Tick-borne diseases and their vectors. Proceedings of an International Conference in Edinburgh, 27 September - 10 October 1976, edited by J.K.H. Wilde, University of Edinburgh: 318-323.

DOLAN, T.T., NJUGUNA, L. \& STAGG, D.A. 1982. The response of Bos taurus and Bos indicus cattle types to inoculation with lymphoblastoid cell lines infected with Theileria parva schizonts. Tropenmedizin und Parasitolgie, 33:57-62.

DOLAN, T.T., YOUNG, A.S., LOGOS, G.J., MCMILLIAN, I., MINDER, C.E. \& SOULSBY, K. 1984. Dose-dependent responses of cattle to Theileria parva stabilate. International Journal for Parasitology, 14:89-95.

JARRET, W.F.H., CRIGHTON, G.W. \& PIRIE, A.M. 1969. Theileria parva: Kinetics of infection. Experimental Parasitology, 24:9-25.

MASON, I.L. \& MAULE, J.P. 1960. The indigenous livestock of East and Central Africa. Farnham Royal, England: Commonwealth Agricultural Bureax.

MOLL, G., LOHDING, A. \& YOUNG, A.S. 1984. Epidemiology of theileriosis in the Trans-Mara Division, Kenya: Husbandry and disease background and preliminary investigations on theileriosis in calves. Preventive Veterinary Medicine, 2:801831.

MOLL, G., LOHDING, A., YOUNG, A.S. \& LEITCH, B.L. 1986. Epidemiology of theileriosis in an endemic area in Kenya. Veterinary Parasitology, 19:55-273.

MORRISON, W.I., BUSCHER, G., MURRAY, M., EMERY, D.L., MASAKE, R.A., COOK, R.M. \& WELLS, P.H. 1981. Theileria parva: Kinetics of infection in the lymphoid system of cattle. Experimental Parasitology, 52:243-260.

MUTUGI, J.J., YOUNG, A.S., MARITIM, A.C.., NDUNGU, S.G., STAGG, D.A., GROOTENHUIS, J.G. \& LEITICH, B.L. 1988. Immunisation of cattle against theileriosis using varying doses of Theileria parva lawrencei and Theileria parva parva sporozoites and oxytetracycline treatment. International Journal for Parasitology, 18:453-461.

RADLEY, D.E., BROWN, C.G.D., BURRIDGE, M.J., CUNNINGHAM, M.P., PEIRCE, M.A. \& PURNELL, R.E. 1974. East Coast fever: Quantitative studies of Theileria parva in cattle. Experimental Parasitology, 36:278-287.

RADLEY, D.E. 1978. Immunisation against East Coast fever by chemoprophylaxis. FAO Technical report 1 AG: DP/RA/67/077. Rome: FAO.

STAGG, D.A., YOUNG, A.S., LEITCH, B., \& DOLAN, T.T. 1983. Infection of mammalian cells with Theileria parva sporozoites in vitro. Parasitology, 86:243-254.

STOBBS, T.N. 1966. The introduction of Boran cattle into an ECF endemic area. East African Agriculture and Forestry Journal, 31:298-308.

YOUNG, A.S. 1981. Epidemiology of theileriosis in East Africa, in Advances in the control of theileriosis, edited by A.D. Irvin, M.P. Cunningham \& A.S. Young. The Hague: Martinus Nijhoff Publishers. 